\title{
Antiinflammatory adaptation to hypoxia through adenosine-mediated cullin-1 deneddylation
}

\author{
Joseph Khoury, ${ }^{1}$ Juan C. Ibla, ${ }^{1}$ Andrew S. Neish, ${ }^{2}$ and Sean P. Colgan ${ }^{3}$ \\ 1Department of Anesthesiology, Perioperative and Pain Medicine, Children's Hospital Boston, Harvard Medical School, \\ Boston, Massachusetts, USA. 'Department of Pathology and Laboratory Medicine, Emory University, Atlanta, Georgia, USA. \\ 3Mucosal Inflammation Program, Division of Gastroenterology, University of Colorado Health Sciences Center, Denver, Colorado, USA.
}

\begin{abstract}
A major adaptive pathway for hypoxia is hypoxic preconditioning (HPC), a form of endogenous protection that renders cells tolerant to severe challenges of hypoxia. We sought to define the antiinflammatory properties of HPC. cDNA microarray analysis of lung tissue from mice subjected to hypoxia or HPC identified a cluster of NF- $\kappa B$-regulated genes whose expression is attenuated by HPC. Studies using an NF- $\kappa B$ luciferase reporter assay confirmed a significant suppression of NF- $\kappa B$ activation during HPC. HPC-elicited activity was conferrable, as a soluble supernatant from HPC-treated cells, and the active fraction was purified and identified as adenosine (Ado). Guided by recent studies demonstrating bacterial inhibition of NF- $\kappa \mathrm{B}$ through cullin-1 (Cul-1) deneddylation, we found a dose-dependent deneddylation of Cul-1 by Ado receptor stimulation predominantly mediated by the Ado A2B receptor subtype. Further, siRNA-mediated repression of CSN5, a subunit of the COP9 signalosome responsible for deneddylation of Cul-1, partially reversed HPC-mediated inhibition of NF- $\kappa \mathrm{B}$. Cul-1 deneddylation was evident in a murine model of HPC and lost in animals lacking extracellular Ado (Cd73-/- mice). Taken together, these results demonstrate that HPC induces extracellular accumulation of Ado and suppresses NF- $\kappa B$ activity through deneddylation of Cul-1. These results define a molecular regulatory pathway by which Ado provides potent antiinflammatory properties.
\end{abstract}

\section{Introduction}

Active inflammatory responses are characterized by significant shifts in tissue metabolism. These changes coincide with increased energy demand and decreased availability of oxygen and can result in significant levels hypoxia (1-3). Thus, it is important to understand the interactions among microenvironmental metabolic changes (e.g., hypoxia) as they relate to the development and resolution of inflammation. Hypoxic preconditioning (HPC) represents a major hypoxia adaptive pathway; however, the molecular mechanisms of HPC remain largely unknown. Recent studies by Zhang et al. (4) have demonstrated that mice subjected to HPC show significant increases in survival when exposed to otherwise lethal levels of hypoxia. Improved lung function was suggested as a major adaptive mechanism, although the ultimate pathways responsible for this effect were not identified.

Regulated protein degradation is an essential feature of cell signaling for many adaptive processes. The proteasomal degrada-

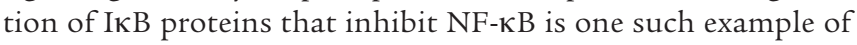
rapid response by the cell to signal for cell growth, differentiation, apoptosis, or inflammation. The E3 SCF ubiquitin ligase specific to IKB family members, comprising SKP1, CUL1, and the F-box domain of $\beta$-TrCP, is responsible for the polyubiquitination of IКB (5). Activity of the E3 ligase requires the regulatory subunit Cul-1 to be posttranslationally modified by the ubiquitin-like protein Nedd8. Loss of the Nedd8 modification is mediated by

Nonstandard abbreviations used: Ado, adenosine; $\mathrm{FiO}_{2}$, fractional inspired $\mathrm{O}_{2}$; HPC, hypoxic preconditioning; IKK $\beta$, IKB kinase; NECA, $N$-ethyl-carbamidoadenosine; 8-PT, 8-phenyltheophylline.

Conflict of interest: The authors have declared that no conflict of interest exists. Citation for this article: J. Clin. Invest. 117:703-711 (2007). doi:10.1172/JCI30049. the COP9 signalosome and results in loss of SCF activity. Deneddylated Cul-1 is incapable of ubiquitination of IKB - hence the inactivation of NF-кB (6).

Physiologic adaptation to hypoxia is an area of intense investigation. In this regard, it is widely accepted that adenosine (Ado) is a critical mediator during ischemia and hypoxia (7) and contributes to conditions as diverse as inflammation and carcinogenesis (8-12). While the source of interstitial Ado in hypoxic tissue has been the basis of much debate, it is now appreciated that inhibition of Ado kinase and the dephosphorylation of ATP and AMP by surface apyrases (e.g., CD39) and ecto-5' -nucleotidase (CD73), respectively, represent the major pathways of extracellular Ado liberation during oxygen supply imbalances (13-15). Once liberated in the extracellular space, Ado is either recycled (e.g., through dipyridamole-sensitive carriers) or interacts with cell-surface Ado receptors (10). Presently, 4 subtypes of G protein-coupled Ado receptors have been identified, designated $\mathrm{A} 1, \mathrm{~A} 2 \mathrm{~A}, \mathrm{~A} 2 \mathrm{~B}$, and $\mathrm{A} 3$, and are classified according to utilization of pertussis toxin-sensitive pathways (A1 and A3) or adenylate cyclase (A2A and A2B) (10). Here, we sought to investigate endogenous mechanisms underlying HPC adaptive responses to hypoxia, particularly as they relate to inflammation.

\section{Results}

$H P C$ attenuates NF- $\mathrm{K} B$ activity via the release of a soluble factor. Initially, we subjected mice to protocols of HPC similar to those of Zhang et al. (4) (see Methods for protocol). Our results confirmed these previous findings and indicated that HPC-adapted animals survive significantly longer when challenged with severe hypoxia (fractional inspired $\mathrm{O}_{2}\left[\mathrm{FiO}_{2}\right] 5 \%$ ) (data not shown). These results led us to define the underlying mechanisms of HPC protection. 
A

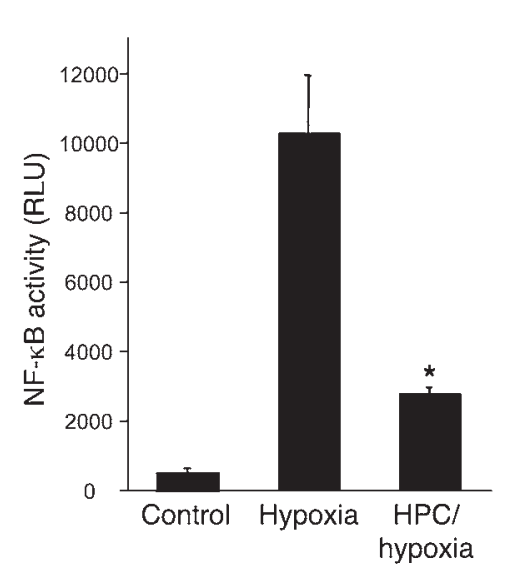

B

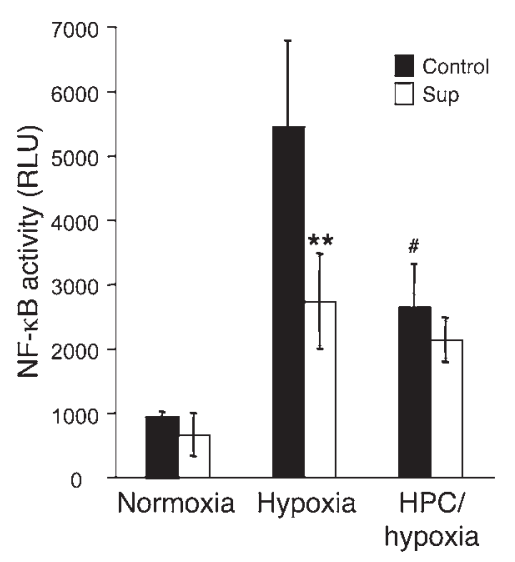

C



Figure 1

HPC attenuates NF-KB activity via the release of a soluble factor. (A) HeLa cells transfected with pNRE were either untreated (Control) or subjected to hypoxia or an HPC protocol followed by hypoxia. Cells that had been preconditioned showed a significant $\left({ }^{\star} P<0.02, \mathrm{HPC}\right.$ versus

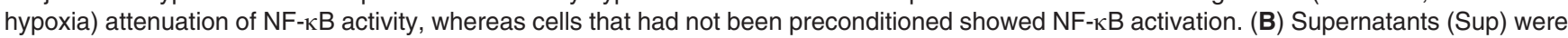
taken from cells undergoing HPC following each hypoxic cycle and were placed on naive cells for 3 cycles of 65 minutes (corresponding to 1 whole preconditioning cycle). Naive cells that received supernatants (white bars) were capable of attenuating NF-kB activity comparably to cells that had been preconditioned $\left({ }^{* *} P<0.05\right.$ supernatant versus control hypoxia; ${ }^{\#} P<0.05$, HPC versus hypoxia). (C) Supernatants taken during HPC were successively filtered through 100-, 30-, and 5-kDa filters and then placed onto naive cells. Filtrates displayed an enhanced inactivation of NF-kB as compared with cells that underwent $\mathrm{HPC}$ alone $\left({ }^{\star} P<0.05\right)$.

To this end, we profiled mRNA transcripts from pulmonary tissue of mice subjected to normoxia, hypoxia, or HPC (see Methods for detailed protocols) using cDNA microarray analysis. This profiling approach identified HPC-dependent repression of a major cluster of NF-кB-mediated inflammatory genes (Supplemental Table 1; supplemental material available online with this article; doi:10.1172/JCI30049DS1). Given the association of NF-KB activation with hypoxia (16), we pursued the antiinflammatory aspects of HPC as they relate to NF-кB.

To define the functional attributes of NF- $\mathrm{BB}$ inhibition by HPC, we used an NF-кB luciferase reporter. HeLa cells were transfected with the pNRE-Luc vector followed by exposure to normoxia, anoxia ( $0 \% \mathrm{O}_{2}, 18$ hours), or HPC followed by anoxia (see HPC protocol in Methods). These studies revealed that cells subjected to HPC displayed a significant attenuation of NF- $\mathrm{\kappa B}$ activation compared with those exposed to anoxia alone (Figure 1A). Likewise, a

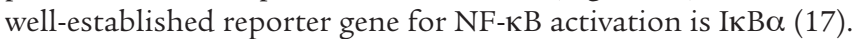
In the course of these experiments, we examined the induction of I $\mathrm{B} \alpha$ by hypoxia and whether such induction was attenuated by HPC. As shown in Supplemental Figure 1, hypoxia was a strong stimulus for induction of IKB $\alpha$ mRNA in HeLa cells (10.4 $\pm 2.0-$ fold increase compared with normoxia control; $P<0.01)$. Parallel examination in HeLa cells subjected to HPC revealed a complete loss of hypoxia-induced IKB $\alpha(0.32 \pm 0.04$-fold change compared with normoxia control; $P<0.01)$, thus confirming our findings from the NF-кB luciferase reporter experiments. Such findings provide in vitro confirmation of our findings in vivo.

We next addressed whether HPC-induced antiinflammation resulted from the release of a transferable factor. Here, we harvested soluble supernatants from HPC-exposed cells and examined hypoxia-

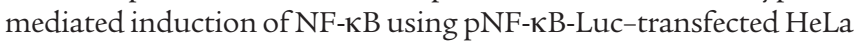
cells. These studies established the existence of a soluble factor that, when transferred to naive cells, confers the HPC phenotype of atten- uated NF-кB activation $(51 \% \pm 5.2 \%$ decrease; $P<0.05$; Figure $1 \mathrm{~B})$. We next used standard biophysical approaches to identify the soluble factor (see Methods). As shown in Figure 1C, a soluble HPC factor of smaller than $5 \mathrm{kDa}$ was evident in supernatants filtered through successively smaller molecular weight cutoffs (i.e., 100-, $30-$, and 5-kDa filters). These results suggested that a small, stable molecule was responsible for the functional attenuation of NF-кB activation by HPC. Spectrometric analysis of the filtrates showed a prominent chromophore at $260 \mathrm{~nm}$ (data not shown), indicating the likely existence of a nucleoside/nucleotide. Further analysis of the active fraction by HPLC revealed high concentrations of Ado within the HPC-conditioned sample (Figure 2A). Moreover, Ado levels increased with successive cycles of HPC (Figure 2B). To determine the role of Ado in the HPC phenotype, we used the nonselective Ado receptor antagonist 8-phenyl-theophylline and demonstrated a significant attenuation in inhibition of NF- $\kappa \mathrm{B}$ by HPC-conditioned supernatants (Figure 2C). These findings of HPC-induced Ado are consistent with previous results implicating Ado in cytoprotection afforded by ischemic preconditioning, particularly in cardiac tissues $(18,19)$. While the source of interstitial Ado in hypoxic tissue has been the basis of much debate, it is now appreciated that inhibition of Ado kinase and the dephosphorylation of AMP by surface apyrases and ecto-5'-nucleotidase (CD73) represent the major pathways of extracellular Ado release during oxygen supply imbalances (13-15). In this regard, Ado and CD73 have been strongly implicated in preconditioning of cardiac tissue $(10,20)$.

Ado analogs inbibit TNF- $\alpha-$ mediated $N F-\kappa B$ activation. Mechanisms of Ado action in inflammatory processes are currently an area of intense investigation (21-23). To extend the findings of HPC-mediated antiinflammation by Ado, we invoked a series of experiments to define mechanisms of Ado action on NF-кB activity. While a number of mechanisms have been suggested to mediate the endogenous inactivation of NF- $\mathrm{KB}$, each of these pathways 

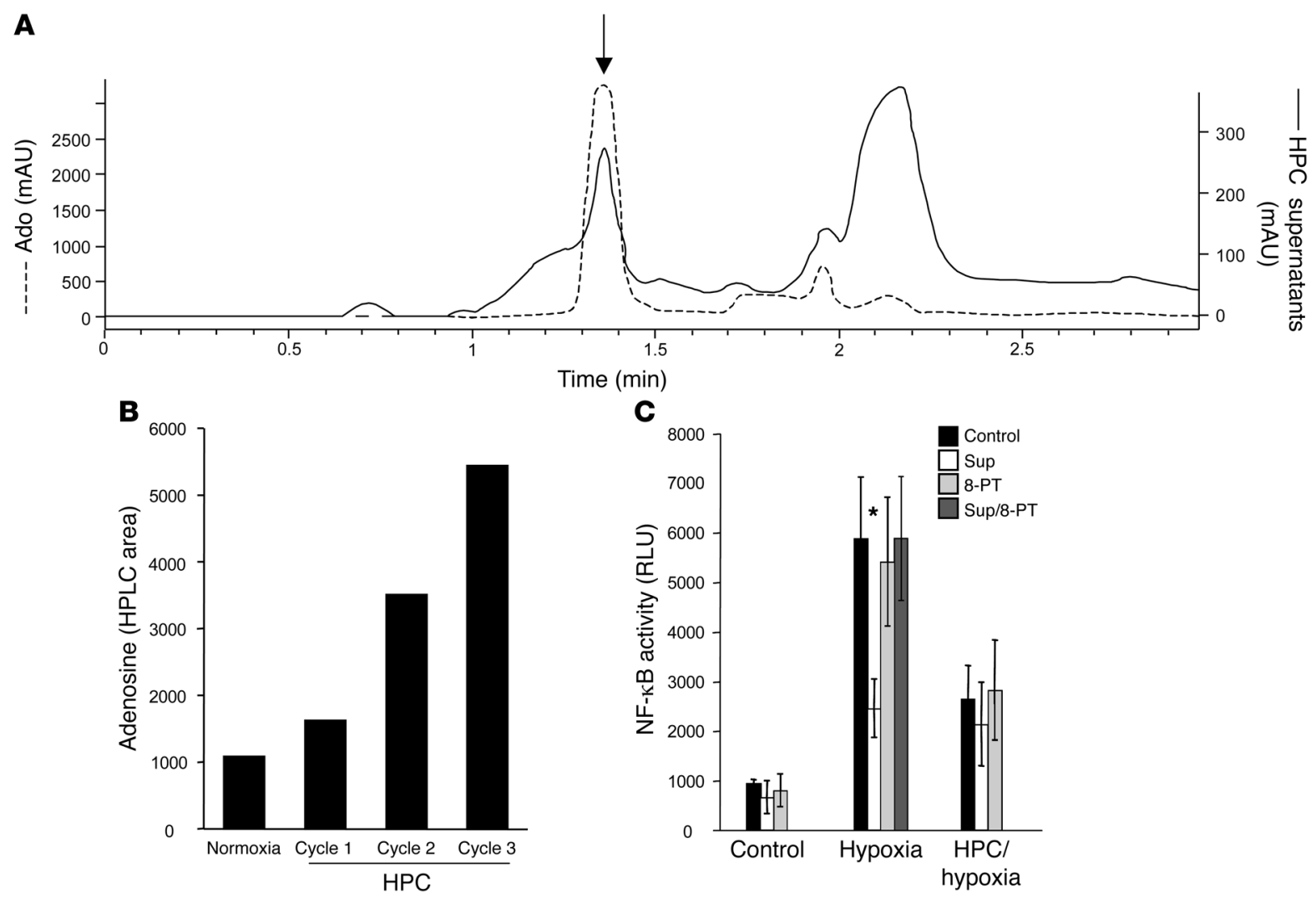

Figure 2

Identification of the HPC soluble factor as Ado. (A) HPLC tracings of the supernatants from preconditioned cells filtered through 5-kDa-MW cutoff filters (solid line, scale on right) display a retention time peak (arrow) identical to an internal standard of Ado (1 mM; dashed line, scale on left). (B) HPLC area of the peak of the filtered supernatant (as in A), showing an increase in Ado production with each cycle of HPC. (C) Incubation of cells with 8-PT during HPC inhibits the release of soluble factor and thus does not allow naive cells the possibility of inhibiting NF- $\kappa B$ activity (dark gray bars, Hypoxia) compared with incubation with supernatants that were not inhibited with 8-PT (white bars; ${ }^{\star} P<0.05$ versus hypoxia alone).

converge on changes in the inducible degradation of $\operatorname{I\kappa B}(24,25)$. Thus, we determined whether Ado influences IкB $\alpha$ stability. For this purpose, HeLa cells were exposed to the stable Ado analog $\mathrm{N}$-ethyl-carbamido-adenosine (NECA), challenged with an NF- $\mathrm{B}$ activator (TNF- $\alpha$ ), and immunoblotted for IкB $\alpha$ (Figure 3A). To obtain additional evidence, we examined the cytoplasmic-to-nuclear translocation of the $\mathrm{p} 65$ subunit NF- $\mathrm{\kappa B}$, an assay commonly used to determine NF- $\mathrm{KB}$ activation. Compared with exposure to TNF- $\alpha$ alone (Figure 3D), treatment with both NECA and TNF- $\alpha$ significantly attenuated TNF- $\alpha$-stimulated p 65 translocation (Figure 3E). No differences in p65 localization were noted between buffer alone and NECA alone (Figure 3, B and C).

Exogenous Ado acts like HPC and results in the deneddylation of Cul-1. In initial studies to define mechanisms of HPC-mediated inhibition of NF- $\kappa \mathrm{B}$, we explored the possibility that Ado release through HPC could lead to inhibition of the IкB kinase (IKK $\beta$ ) and thus result in the loss of IK $B \alpha$ phosphorylation as a mechanism of $\mathrm{NF}-\kappa \mathrm{B}$ inhibition. To address this issue, protein lysates from HeLa cells exposed to either normoxia, hypoxia, or HPC were immunoblotted for total IKK $\beta$ as well as phospho-IKK $\beta$. As shown in Figure 3F, no significant differences were observed in IKK $\beta$ expression between cells exposed to hypoxia and HPC, suggesting that cells subjected to HPC normally activate IKK $\beta$ and therefore maintain a normal phosphorylation mechanism for IкB $\alpha$.
Recent studies identified a mechanism of inhibition of NF- $\mathrm{KB}$ by probiotic bacteria (26). These studies implicated the active deneddylation of Cul-1 by bacteria and its direct inactivation of NF- $\kappa B$. Importantly, this cullin-1 deneddylation occurs in the presence of normal IкB $\alpha$ phosphorylation patterns (26), such as we observed with HPC. These findings with probiotic bacteria were based on the fact that ubiquitination of phosphorylated IкB occurs through interactions with the SCF E3 ubiquitin ligase complex (consisting of Skp1, Cul-1, and $\beta$-TrCP F-box) (24). This SCF complex is active only when Cul-1 is covalently modified by the ubiquitin-like protein Nedd8, and, hence, the neddylation status of Cul-1 directly reflects NF-KB activation (27). Based on these previous studies, we examined whether Ado receptor activation influenced the neddylation of Cul-1. As shown in Figure 4A, immunoblotting of Cul-1 derived from HeLa cell lysates revealed a higher-molecular-weight band, which has been previously shown to be the neddylated form of Cul-1 (26). Densitometric analysis of these results are shown in Supplemental Figure 2A. An immunoblot directed against the Nedd8 protein revealed a pattern similar to the upper band pattern seen for Cul-1, further demonstrating the presence of Nedd 8 modification on Cul-1. Indeed, an NECA concentration-dependent loss of Nedd8 was apparent by Western blot analysis (Figure 4A).

We next demonstrated that these findings are not specific for HeLa cells. Here we used CaLu-3 cells, a lung cell line, for purpos- 
A



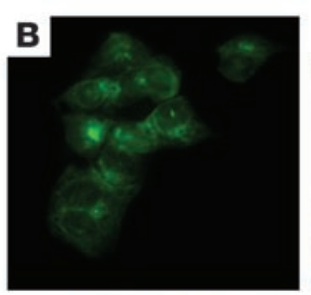

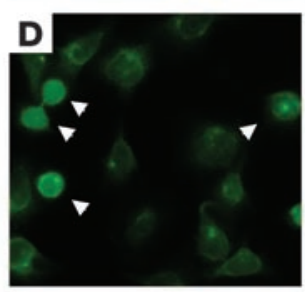

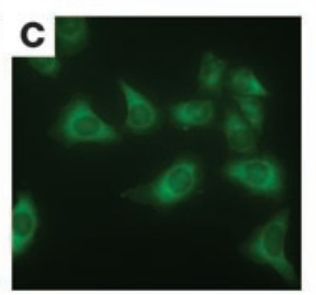

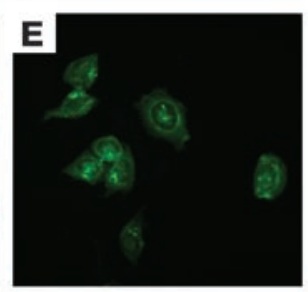



\section{Figure 3}

Ado analogs (NECA) inhibit TNF- $\alpha-$ mediated NF-кB activation. (A) Western blot analysis of the stabilization of I $\mathrm{kB} \alpha$. While NECA stimulation


stabilization of $\mathrm{I}_{\kappa} \mathrm{B} \alpha$ was reestablished when cells were pretreated with NECA prior to TNF- $\alpha$ stimulation. Similarly, NECA inhibited TNF- $\alpha-$ mediated p65 nuclear translocation, as shown in D (arrowheads). (B) Control conditions. (C) NECA stimulation (10 $\mu$ M; 30 minutes). (D) TNF- $\alpha$ stimulation ( $3 \mathrm{ng} / \mathrm{ml}$; 15 minutes). (E) NECA pretreatment ( $10 \mu \mathrm{M}, 30$ minutes) followed by TNF- $\alpha$ stimulation ( $3 \mathrm{ng} / \mathrm{ml} ; 15 \mathrm{minutes})$. Original magnification, $\times 400$. (F) Representative Western blot of total and phospho-specific IKK $\beta$ in HeLa cells subjected to normoxia, hypoxia, and HPC.

es of comparison. As shown in Figure 4B, CaLu-3 cell Cul-1 was similarly deneddylated by NECA when probed for Nedd8 by Western blotting, suggesting that this finding is not limited to HeLa cells. Ado receptor stimulation with NECA revealed a concentration-dependent deneddylation of Cul-1, with an $\mathrm{EC}_{50}$ of approximately $30 \mathrm{nM}$. Densitometric analysis of these results are shown in Supplemental Figure 2B. Moreover, the general Ado receptor antagonist 8-phenyltheophylline (8-PT) potently inhibited NECAinduced deneddylation (Figure 4C).

To define Ado receptor subtype specificity, we utilized heterologous overexpression of individual Ado receptors in HeLa cells, wherein cells were cotransfected with pNF-кB-Luc and examined for inhibition by HPC. As shown in Figure 4D, the Ado receptor determining HPC-mediated inhibition of pNF-кB-Luc activity was predominantly $\mathrm{A} 2 \mathrm{~B}(P<0.01)$ and to a lesser extent A1 receptors $(P<0.05)$.

siRNA-mediated repression of the CSN5 component of the COP9 signalosome inhibits Cul-1 deneddylation. Deneddylation of Cul-1 is normally accomplished through interaction with subunits of the COP9 signalosome (e.g., subunit 5 or CSN5/JAB1) (27). Here, we examined whether repression of CSN5 expression (and thereby inhibition of Cul-1 deneddylation) might influence the antiinflammatory aspects of HPC (i.e., attenuation of NF-кB activation). For these purposes, we transfected HeLa cells with siRNA directed against CSN5/JAB1 and examined Ado and HPC influences on Cul-1 deneddylation and NF-кB activation, respectively. As shown in Figure 4E, this siRNA strategy decreased CSN5 protein expression by greater than $70 \%$ relative to mock transfection. Under these conditions, NECA-induced Cul-1 deneddylation was nearly completely abolished compared with control (Figure 4E), and CSN5-repressed cells lost their ability to precondition with respect to hypoxia-induced NF- $\kappa \mathrm{B}$ activation (Figure 4F). These findings confirm our hypothesis that HPC confers an antiinflammatory phenotype through influences on CSN5-mediated neddylation of Cul-1.

HPC offers in vivo protection to mice by decreasing inflammation in lungs and by deneddylating $\mathrm{Cul}-1$. In a proof of principle experiment examining whether Ado production contributes to deneddylation of Cul-1 in vivo, we compared wild-type with $\mathrm{Cd} 73^{-/-}$null mice, which lack the critical enzyme for extracellular Ado formation (ecto-5'-nucleotidase or CD73) (28). Cd73 ${ }^{-/}$or wild-type mice were subjected to protocols of preconditioning followed by hypoxia and lung tissues harvested for analysis. Figure 5A shows explanted whole left lungs from mice exposed to protocols of HPC and revealed that lungs from wild-type animals subjected to hypoxia alone were large and swollen, indicative of significant hemorrhage and edema. Lungs from wild-type animals that had been preconditioned had the macroscopic appearance of normoxic controls. By contrast, lungs of the $\mathrm{Cd} 73^{-/}$mice displayed increased volume in all but the normoxic condition, indicating a nearly complete loss of HPC phenotype in Cd73-/- mice (Supplemental Figure 5). Histology of the left lung from hypoxic animals revealed notable perivascular hemorrhage located in the alveolar spaces, whereas the normoxic and preconditioned mice showed no signs of hemorrhage (Figure 5, B-G). Hemorrhage consists of not only red blood cells, but also plasma, which contributes to edema. Further, periarterial cuffing was observed (Figure 5, H-J) similar to that originally identified by Whayne and Severinghaus in acute hypoxia in rats (29). Hypoxia often leads to both inflammation and edema in the lung; therefore, we used edema formation (tissue water content) as a marker of inflammation and barrier function. To further examine this observation, we measured lung water content as an endpoint for evolving inflammation. As shown in Figure 6A, in wild-type mice, hypoxia alone led to an increase in lung water when compared with normoxia or HPC $(P<0.02)$. However, lungs of $\mathrm{Cd} 73^{-/}$mice had an increased level of lung water and showed no significant decrease with HPC, thereby confirming our macroscopic observations (Figure 5A and Supplemental Figure 4). Extensions of these findings revealed that mice subjected to protocols of HPC followed by hypoxia display deneddylate Cul-1 in immunoblots from protein derived from lung tissue (Figure 6B). Densitometric analysis of these results are shown in Supplemental Figure 3. Interestingly, in whole lung tissue in vivo, hypoxia increased the ratio of neddylated to unmodified Cul-1, thereby implicating the activation of NF- $\mathrm{\kappa B}$ due to an increase in 
A



D $\quad$ E

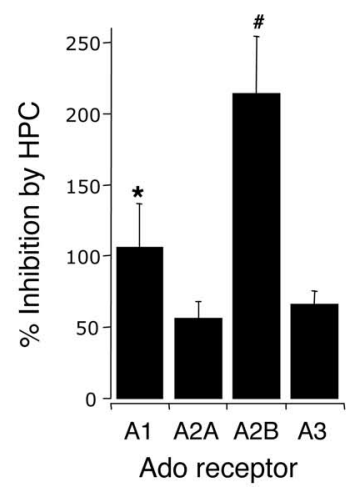

B

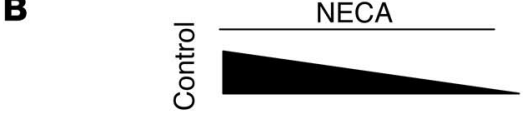

Nedd8 -

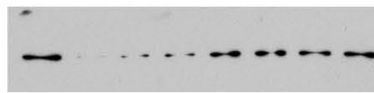

Actin -

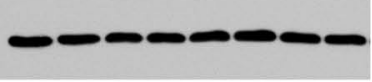

C



E

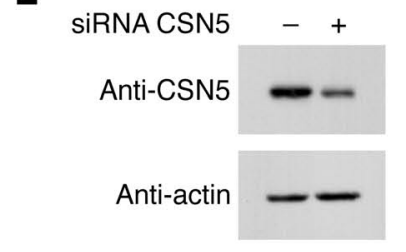

$\mathbf{F}$

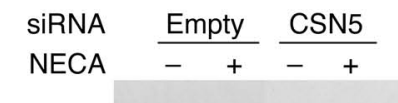

Cul-1 ${ }^{\text {NEDD8 }}$

Cul-1-

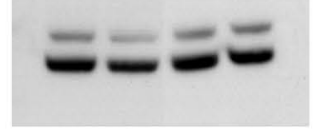

G

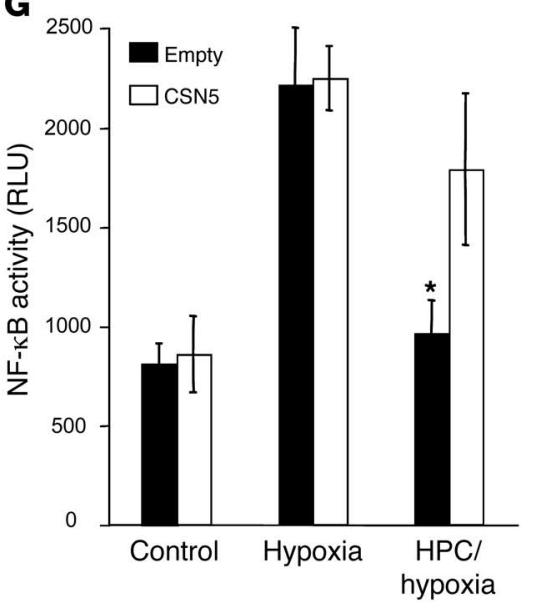

Figure 4

Exogenous Ado acts like HPC and results in the deneddylation of Cul-1, and siRNA-mediated repression of the CSN5 component of the COP9 signalosome inhibits Cul-1 deneddylation. Antibodies against Cul-1 reveal 2 bands: a lower unmodified Cul-1 protein and an upper, neddylated form, Cul-1 NEDD8. HeLa cell lysates were prepared as described in Methods, separated on 8\% SDS-PAGE, and blotted against Cul-1, Nedd8, or actin (A-C). Log dose response of NECA (100 $\mu \mathrm{M}$ to $100 \mathrm{pM} ; 30$ minutes) in HeLa (A) or CaLu-3 cells (B) shows potent deneddylation of Cul-1. Deneddylation could be seen with as little as $1 \mathrm{nM} \mathrm{NECA}$; $\mathrm{EC}_{50}$ is estimated to be $30 \mathrm{nM}$. (C) Preincubation with 8-PT (10 nM; 15 minutes) inhibits the deneddylation effect on Cul-1 by NECA at both $100-\mu \mathrm{M}$ and $10-\mu \mathrm{M}$ doses (30 minutes). (D) Inhibition by HPC in cells overexpressing individual Ado receptors as compared to cells expressing endogenous levels of Ado receptors and examined for inhibition of NF- $\kappa B$. Data are expressed as percent inhibition of NF-KB activation \pm SEM for individual receptors; ${ }^{*} P<0.05, \# P<0.01$. (E) siRNA targeted against the CSN5 component of the COP9 signalosome results in approximately $70 \%$ reduction in protein levels when blotted against a CSN5 antibody. (F) NECAmediated (10 mM; 30 minutes) deneddylation of Cul-1 was inhibited in cells with knockdown of CSN5 (siCSN5) compared with cells that had not been knocked down (Empty). (G) CSN5 knockdown of cells (white bars, siCSN5) does not affect NF-kB activity in control or hypoxic cells, however, it causes HPC/hypoxia cells to lose their ability to attenuate NF-kB. ${ }^{*} P<0.05, \mathrm{HPC} /$ hypoxia versus hypoxia alone.

neddylation by hypoxia. Conversely, HPC resulted in a near complete loss of Cul-1 neddylation, consistent with NF-кB repression. Similarly, IкB immunoblots confirmed the increased levels of $\mathrm{NF}-\kappa \mathrm{B}$ in hypoxic lungs and the apparent decrease with HPC, whereas the lungs of the $\mathrm{Cd} 73^{-/-}$mice did not show such changes. Taken together, such findings of directed deneddylation by Ado and HPC suggest an active inhibitory loop for NF-KB activation. Notably, Western blots from lung tissue from $\mathrm{Cd} 73^{-/-}$mice displayed hyperneddylated Cul-1 and further showed the loss of deneddylation with HPC observed in wild-type animals. This observation thereby confirms our findings that Ado may play an important role in the stabilization of the E3 SCF ubiquitin ligase and its role in the inactivation of NF-KB.

\section{Discussion}

Inflammation can be defined as a complex set of interactions among soluble factors and cells that arise in any tissue in response to traumatic, infectious, postischemic, toxic, or autoimmune injury (30). The inflammatory process normally leads to recovery; however, if not properly phased, it could lead to tissue damage. Inflammation is commonly seen in many pulmonary disorders, including high altitude pulmonary edema (31) and hypoxiainduced pulmonary hypertension (32).

Inflammatory pathways are regulated by a limited number of transcription factors, the most important being NF-кB (33). Upon cellular stimulation by a variety of mediators, including cytokines, bacterial toxins, or oxidative stress, a signal transduction cascade is activated, leading to the phosphorylation of IкB $\alpha$ on Ser32 and -36 by the multimeric IKK complex (34). Phosphorylation of IкB $\alpha$ is followed by ubiquitination via the E3 ligase SCF $\beta^{\mathrm{TRCP}}$ and is targeted for proteasomal degradation by the $26 \mathrm{~S}$ proteasome (35).

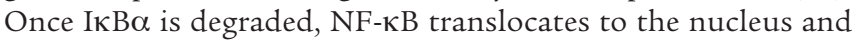
binds to the promoter regions of several proinflammatory genes, inducing their expression and thus amplifying the inflammatory response. The extent of NF- $\mathrm{KB}$ activation depends on diverse factors, including the variable E3 activity of the SCF $\beta^{\text {TRCP }}$ complex, which is regulated by a reversible covalent modification with the ubiquitin-like protein Nedd8 (36). 

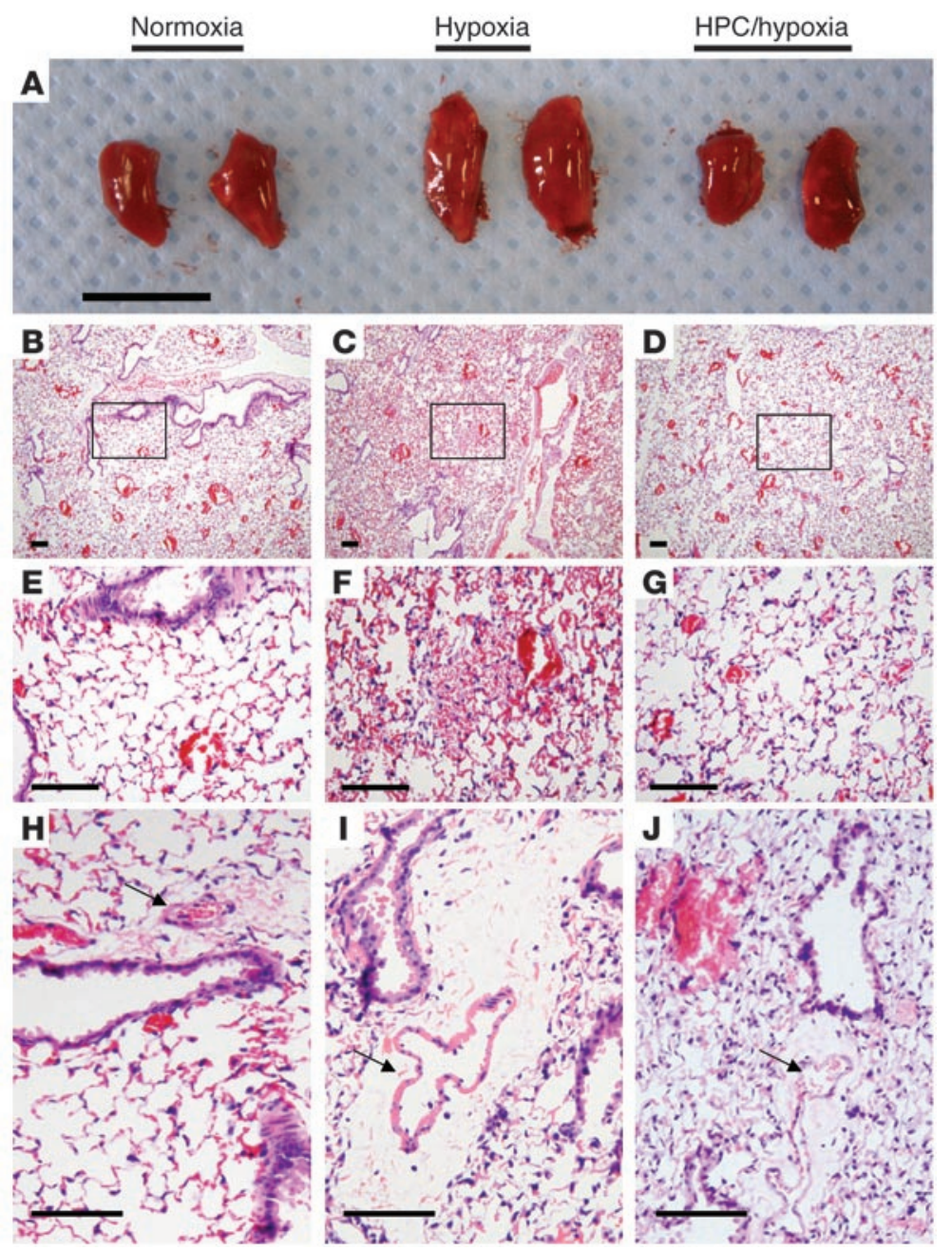

Figure 5

HPC offers in vivo protection to mice by decreasing inflammation in lungs. (A) Whole left lungs from mice were harvested following protocols of normoxia; severe hypoxia; HPC followed by severe hypoxia. Wild-type lungs display a macroscopic increase in tissue volume only in hypoxic conditions. Scale bar: $1 \mathrm{~cm}$. Histology of the lungs shows noticeable hemorrhage in the hypoxic condition, while the lungs of preconditioned mice display histology similar to that of control, normoxic lungs. Scale bar: $50 \mu \mathrm{m}$. (B-D) Low-power magnification. (E-J) Higher-power magnification shows normoxic histology ( $\mathbf{B}$ and $\mathbf{E}$ ) and normal artery size (arrow) and periarterial space. Hypoxic lung displaying perivascular hemorrhage ( $\mathbf{C}$ and F) and dilated artery and periarterial cuffing (I). HPC followed by hypoxia (D, G, and $\mathbf{J}$ ) showing histology similar to that in normoxia.

In the current study, we have assessed the role of Ado as an antiinflammatory molecule released during HPC. Our studies revealed that cells subjected to HPC display a significant attenuation of NF- $\kappa \mathrm{B}$ activation compared with those exposed to anoxia alone. A soluble mediator found to be released during HPC was identified as Ado. These findings are consistent with our findings in vivo that lungs of mice subjected to HPC prior to more severe hypoxia display no signs of perivascular hemorrhage, a precursor to NF- $\kappa \mathrm{B}$-mediated inflammation $(37,38)$.

A recent study by Majumdar and Aggarwal (39) identified Ado as a suppressor of NF- $\mathrm{KB}$ activity induced by TNF- $\alpha$ and concluded that it may contribute to its role in suppression of inflamma- tion of the immune system. We further showed that Ado analogs such as NECA are capable of inhibiting NF- $\kappa \mathrm{B}$ activation as measured by I $\mathrm{B} \alpha$ degradation by the potent NF- $\kappa$ B activator TNF- $\alpha$. The exact mechanism

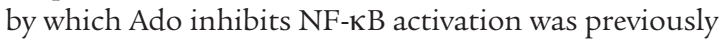
unknown. Our studies focused on one pathway involved in the regulation of NF- $\mathrm{KB}$ activation, the ubiquitination

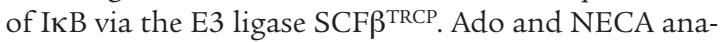
logs displayed a dose-dependent deneddylation of Cul-1 (Figure 4A). It has been demonstrated that the SCF complex is active only when Cul-1 is covalently modified by the ubiquitin-like protein Nedd8; therefore, the neddylation status of Cul-1 directly reflects NF- $\mathrm{\kappa B}$ activation (40). We further examined not only the neddylation status of Cul-1 but also, by direct immunoblotting against Nedd8, whether these effects were inhibited by the general Ado receptor antagonist 8-PT.

The COP9 signalosome (CSN), an 8-subunit complex that regulates protein ubiquitination, posttranscriptionally modifies the cullin subunit of E3-ubiquitin ligases by cleaving off the covalently coupled protein Nedd8 $(5,6)$. Of the 8 different subunits of CSN, CSN5/ JAB1, which has metalloprotease activity, has been established to be important in the role of deneddylating Cul-1. The cleavage of the Nedd 8 moiety is catalyzed not by a conventional thiol protease, but rather by metalloisopeptidase activity centered within the CSN5 subunit (5). In our studies, downregulation of CSN5/JAB1 by siRNA inhibited NECA-induced Cul-1 deneddylation and resulted in a loss of preconditioning as measured by NF- $\kappa \mathrm{B}$ activation (Figure 4, E and F). These findings confirm the role of Ado released during HPC in conferring an antiinflammatory phenotype through influences on CSN5-mediated neddylation of Cul-1.

The findings were tested in vivo to further elucidate the pathway by which Ado released during HPC confers antiinflammatory protection via NF- $\mathrm{KB}$ inhibition. Our in vivo findings are consistent with those of in vitro studies, particularly those showing that Cul-1 is deneddylated following HPC and that stabilization of IкB is maintained. Morphologically and histologically, evidence of antiinflammation was indicated by the gross size and water content of the lung as well as the protection conferred against hemorrhage. Hemorrhage has been well established to be a precursor to NF-KB-mediated inflammation $(37,38)$. Hemorrhage consists of not only red blood cells, but also plasma, which contributes to pulmonary edema. H\&E staining did not reveal plasma infiltration in the alveolar spaces, but edema was evidenced in the wet/dry ratio. With regard to the direct role of NF-кB inhibition in this model, it is difficult to assess the exact contribution of Ado. For example, a number of drugs that have been shown to inhibit NF-кB activation (e.g., pyrrolidine dithiocarbamate) can result in tissue phenotypes and outcomes that closely resemble HPC (41). However, none of the known NF-кB inhibitors used in in vivo settings have sufficient specificity to convincingly distinguish the role of NF- $\mathrm{NB}$ in preconditioning. A recent study has shed new light on this topic. Frantz et al. utilized NF-кB p50 subunit knockout mice to investigate the role of NF- $\mathrm{KB}$ in myocardial preconditioning (42). These studies revealed a crucial role for p50 


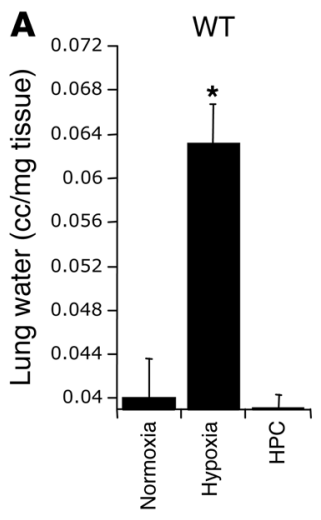

B

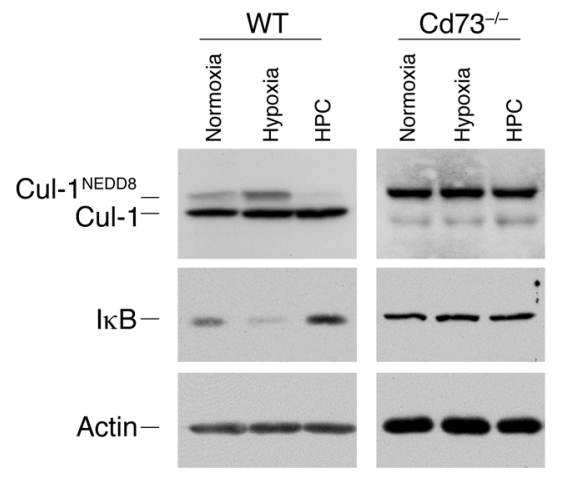

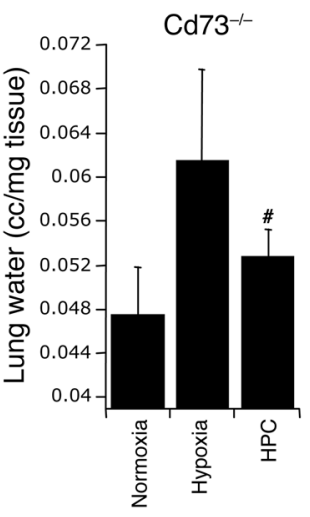

$$
\text { (1) }
$$


cDNA was arrayed through the Massachusetts General Hospital DNA Core Facility using the PGA mouse v1.1 chip. For each experiment, 3 animals were arrayed in triplicate.

Cell culture, treatments, transfections, and in vitro preconditioning. HeLa cells (ATCC) were maintained in DMEM plus $10 \% \mathrm{FBS}$ at $37^{\circ} \mathrm{C}$ in a humidified incubator with $5 \% \mathrm{CO}_{2}$ in room air. Where indicated, cells were treated with (all chemicals from Sigma-Aldrich, unless otherwise noted) NECA in DMSO at concentrations varying from $100 \mathrm{pM}$ to $100 \mu \mathrm{M}$; Ado in $\mathrm{H}_{2} \mathrm{O}, 1 \mathrm{nM}$ to $100 \mu \mathrm{M}$; 8-PT in PBS, $1 \mu \mathrm{M}$; or TNF- $\alpha$ (R\&D Systems) in PBS, $3 \mathrm{ng} / \mathrm{ml}$. Treatment time is indicated in results. Transfection of cells was carried out in 24-well plates using PolyFect reagent as directed by the manufacture (QIAGEN). Plasmids used in transfections were PNF-KB-Luc $(0.4 \mu \mathrm{g} /$ well; Clontech $)$ or each individual Ado receptor in pCMV6 (OriGene) along with Renilla ( $2 \mathrm{ng} /$ well). Cellular preconditioning was performed on cells that were plated on either 6- or 24-well plates following a modified in vivo protocol optimized for cells (4). HeLa cells were placed in a hypoxia chamber (Coy Laboratory Products Inc.) in preequilibrated hypoxic HBSS at $2 \% \mathrm{O}_{2}$ for 45 minutes. Cells were returned to normoxic conditions $\left(21 \% \mathrm{O}_{2}\right)$ for 20 minutes. When cells were returned to hypoxia, media was once again replaced with fresh hypoxic HBSS in order to minimize the effects of oxygen present. This protocol was followed for 3 cycles, and then, where indicated, cells were placed in an anoxia chamber $\left(0 \% \mathrm{O}_{2}\right)$ with fresh preequilibrated anoxic HBSS for the times indicated.

$N F-\kappa B$ reporter assays. To measure transcriptional activity of NF- $\kappa \mathrm{B}, \mathrm{HeLa}$ cells in 24-well plates were transfected with pNF-кB-Luc (pNRE; Clontech) at a concentration of $0.4 \mu \mathrm{g}$ per well along with $0.02 \mu \mathrm{g}$ of Renilla for 24 hours and then either left at normoxia or subjected to a protocol of preconditioning $\left(2 \% \mathrm{O}_{2}\right.$ for 45 minutes followed by $21 \% \mathrm{O}_{2}$ for 20 minutes for 3 cycles; fresh hypoxic media was changed prior to the start of each hypoxic interval) and were then either treated with $3 \mathrm{ng} / \mathrm{ml}$ TNF- $\alpha$ or subjected to anoxia $\left(0 \% \mathrm{O}_{2}\right)$ for an additional 6-24 hours. In experiments where supernatants were studied, supernatants were collected from an additional group of cells following intervals of HPC and placed on normoxic cells for 65 minutes (3 cycles). Following treatments, cells were rinsed in PBS, lysed in passive lysis buffer (Promega) for 15 minutes, and spun down, and $20 \mu$ l of lysates was assayed using the Dual-Glo Luciferase assay system (Promega) with the use of a luminometer (Turner BioSystems).

Analysis of soluble factor. HeLa cells plated in 6-well plates were subjected to preconditioning, and supernatants were collected following each hypoxic event and replaced with fresh hypoxic media at the start of the next cycle. Supernatants were systematically analyzed according to size (filtered through 100-, 30-, and 5-kDa filters), stability (heat, freeze/thaw cycles), and UV chromophores. In each case, the fractions were placed on HeLa cells transfected with pNF-кB-Luc in 24-well plates and assayed as described above. Further analyses of the active fraction by HPLC identified the molecule as Ado and were confirmed by coinjection with internal standards.

Transcriptional analysis. Real-time PCR (iCycler; Bio-Rad) was employed to examine IкB $\alpha$ expression levels in HeLa cells. Primer sets contained $10 \mathrm{pM}$ each of the sense primer 5'-AACCTGCAGCAGACTCCACT-3' and the antisense primer 5'-AGTCTCGGAGCTCAGGATCA-3'. The primer set was amplified using increasing numbers of cycles of $94^{\circ} \mathrm{C}$ for 1 minute, $60^{\circ} \mathrm{C}$ for 2 minutes, $72^{\circ} \mathrm{C}$ for 4 minutes, and a final extension of $72^{\circ} \mathrm{C}$ for 7 minutes. Samples were controlled for $\beta$-actin using the following primers: sense 5'-GGTGGCTTTTAGGATGGCAAG-3'; antisense 5'-ACTGGAACGGTGAAGGTGACAG-3'; 162 bp).

Western blot and immunofluorescence analyses. Whole-cell lysates were isolated from HeLa cells using reducing buffer (62.5 mM Tris $\mathrm{pH}$ 6.8, 2\% SDS, 10\% glycerol, $5 \% \beta$-mercaptoethanol, $1 \%$ bromophenol blue). An equal number of cells were lysed and boiled. The samples were then resolved on $8 \%$ polyacrylamide denaturing gels and transferred to nitrocellulose (Bio-Rad). After transfer, the membranes were stained with ponceau $\mathrm{S}$ stain in order to verify equal loading. Antibodies used for Western blotting included rabbit polyclonal anti-Cul-1 (1:200; Zymed) and goat polyclonal anti-actin (1:1,000; Santa Cruz Biotechnology Inc.). Blots were washed, and species-matched peroxidase-conjugated secondary antibody was added. Labeled bands from washed blots were detected by enhanced chemiluminescence (Amersham Biosciences). For p65 nuclear translocation studies, HeLa cells were plated on 4-well glass chamber slides (Nalgene Nunc International) and allowed to grow to approximately $70 \%$ confluence. Treatment with NECA and or TNF- $\alpha$ was as described above. Cells were fixed in $1 \%$ paraformaldehyde/PBS at $4{ }^{\circ} \mathrm{C}$ for 10 minutes and permeabilized with prechilled $0.2 \%$ Triton X-100/PBS/2\% BSA. Cells were incubated with rabbit anti-p65 (1:200; Rockland Immunochemicals) in 1\% normal goat serum in PBS for 1 hour followed by anti-rabbit Oregon Green 514 (1:100, Molecular Probes; Invitrogen) in the same buffer for 30 minutes. Cell images were captured on a fluorescence microscope.

Tissue water content. Whole left lungs were harvested from wild-type or Cd73 $/-$ mice following HPC protocols and were photographed for macroscopic assessment of inflammation. Lungs were then placed in preweighed Eppendorf tubes and placed in a SpeedVac (Thermo Scientific) set for vacuum mode at $65^{\circ} \mathrm{C}$ for 12 hours. Dry weight was then subtracted from the wet weight to calculate cc water per milligram tissue (51).

Statistics. Values are expressed as mean \pm SEM. Where appropriate, groups were compared by analysis of variance (ANOVA), otherwise, comparisons between groups were conducted using 2-tailed Student's $t$ test. A $P$ value of less than 0.05 was considered to be significant.

\section{Acknowledgments}

This work was supported by a grant from the Foundation for Anesthesia Education and Research (FAER) to J.C. Ibla; and NIH grants HL60569, DK50189, and DE13499 to S.P. Colgan.

Received for publication August 13, 2006, and accepted in revised form January 2, 2007.

Address correspondence to: Juan C. Ibla, Department of Anesthesiology, Perioperative and Pain Medicine, Children's Hospital, Bader 3, 300 Longwood Avenue, Boston, Massachusetts 02115, USA. Phone: (617) 355-7737; Fax: (617) 730-0894; E-mail: juan.ibla@childrens.harvard.edu.

Joseph Khoury and Juan C. Ibla contributed equally to this work.
1. Haddad, J.J. 2003. Science review: redox and oxygen-sensitive transcription factors in the regulation of oxidant-mediated lung injury: role for hypoxiainducible factor-1alpha. Crit. Care. 7:47-54.

2. Kokura, S., Yoshida, N., and Yoshikawa, T. 2002. Anoxia/reoxygenation-induced leukocyteendothelial cell interactions. Free Radic. Biol. Med. 33:427-432.

3. Saadi, S., Wrenshall, L.E., and Platt, J.L. 2003. Regional manifestations and control of the immune system. FASEB J. 16:849-856.

4. Zhang, S.X., Miller, J.J., Gozal, D., and Wang, Y. 2004. Whole-body hypoxic preconditioning protects mice against acute hypoxia by improving lung function. J. Appl. Physiol. 96:392-397.

5. Cope, G.A., and Deshaies, R.J. 2003. COP9 signalosome: a multifunctional regulator of SCF and other cullin-based ubiquitin ligases. Cell. 114:663-671.

6. Cardozo, T., and Pagano, M. 2004. The SCF ubiquitin ligase: insights into a molecular machine. Nat.
Rev. Mol. Cell Biol. 5:739-751.

7. Ohta, A., and Sitkovsky, M. 2001. Role of G-protein-coupled adenosine receptors in downregulation of inflammation and protection from tissue damage. Nature. 414:916-920.

8. Fishman, P., Bar-Yehuda, S., Madi, L., and Cohn, I 2002. A3 adenosine receptor as a target for cancer therapy. Anticancer Drugs. 13:437-443.

9. Li, S., Huang, S., and Peng, S.B. 2005. Overexpression of $\mathrm{G}$ protein-coupled receptors in cancer cells: 
involvement in tumor progression. Int. J. Oncol. 27:1329-1339.

10. Linden, J. 2001. Molecular approach to adenosine receptors: receptor-mediated mechanisms of tissue protection. Annu. Rev. Pharmacol. Toxicol. 41:775-787.

11. Merighi, S., et al. 2003. A glance at adenosine receptors: novel target for antitumor therapy. Pharmacol. Ther. 100:31-48.

12. Sitkovsky, M., and Lukashev, D. 2005. Regulation of immune cells by local-tissue oxygen tension: HIF1 alpha and adenosine receptors. Nat. Rev. Immunol. 5:712-721.

13. Decking, U.K., Schlieper, G., Kroll, K., and Schrader, J. 1997. Hypoxia-induced inhibition of adenosine kinase potentiates cardiac adenosine release. Circ. Res. 81:154-164.

14. Eltzschig, H.K., et al. 2003. Coordinated adenine nucleotide phosphohydrolysis and nucleoside signaling in posthypoxic endothelium: role of ectonucleotidases and adenosine $\mathrm{A}_{2 \mathrm{~B}}$ receptors. J. Exp. Med. 198:783-796.

15. Synnestvedt, K., et al. 2002. Ecto-5'-nucleotidase (CD73) regulation by hypoxia-inducible factor-1 (HIF-1) mediates permeability changes in intestinal epithelia. J. Clin. Invest. 110:993-1002. doi:10.1172/ JCI200215337.

16. Cummins, E.P., and Taylor, C.T. 2005. Hypoxiaresponsive transcription factors. Pflugers Arch. 450:363-371.

17. Algarte, M., Kwon, H., Genin, P., and Hiscott, J. 1999. Identification by in vivo genomic footprinting of a transcriptional switch containing NF-kappaB and $\mathrm{Sp} 1$ that regulates the IkappaBalpha promoter. Mol. Cell. Biol. 19:6140-6153.

18. Baxter, G.F. 2002. Role of adenosine in delayed preconditioning of myocardium. Cardiovasc. Res. 55:483-494.

19. Mubagwa, K., and Flameng, W. 2001. Adenosine, adenosine receptors and myocardial protection: an updated overview. Cardiovasc. Res. 52:25-39.

20. de Jonge, R., Bradamante, S., and de Jong, J.W. 1998. Cardioprotection by ischemic preconditioning. Role of adenosine and glycogen. Adv. Exp. Med. Biol. 431:279-282.

21. Hasko, G., and Cronstein, B.N. 2004. Adenosine: an endogenous regulator of innate immunity. Trends Immunol. 25:33-39.

22. Linden, J. 2005. Adenosine in tissue protection and tissue regeneration. Mol. Pharmacol. 67:1385-1387.

23. Sitkovsky, M.V., et al. 2004. Physiological control of immune response and inflammatory tissue damage by hypoxia-inducible factors and adenosine A2A receptors. Annu. Rev. Immunol. 22:657-682.

24. Chen, Z.J. 2005. Ubiquitin signalling in the NFkappaB pathway. Nat. Cell Biol. 7:758-765.

25. Luo, J.L., Kamata, H., and Karin, M. 2005. IKK/NF-кB signaling: balancing life and death - a new approach to cancer therapy. J. Clin. Invest. 115:2625-2632. doi:10.1172/JCI26322.

26. Collier-Hyams, L.S., Sloane, V., Batten, B.C., and Neish, A.S. 2005. Cutting edge: bacterial modulation of epithelial signaling via changes in neddylation of cullin-1. J. Immunol. 175:4194-4198.

27. Mikus, P., and Zundel, W. 2005. COPing with hypoxia. Semin. Cell Dev. Biol. 16:462-473.

28. Thompson, L.F., et al. 2004. Crucial role for ecto$5^{\prime}$-nucleotidase (CD73) in vascular leak during hypoxia. J. Exp. Med. 200:1395-1405.

29. Whayne, T.F., Jr., and Severinghaus, J.W. 1968. Experimental hypoxic pulmonary edema in the rat. J. Appl. Physiol. 25:729-732.

30. Nathan, C. 2002. Points of control in inflammation. Nature. 420:846-852.

31. Bartsch, P., Mairbaurl, H., Maggiorini, M., and Swenson, E.R. 2005. Physiological aspects of high-altitude pulmonary edema. J. Appl. Physiol. 98:1101-1110.

32. Basnyat, B., and Murdoch, D.R. 2003. High-altitude illness. Lancet. 361:1967-1974.

33. Barnes, P.J., and Karin, M. 1997. Nuclear factor-кB - a pivitol transcription factor in chronic inflammatory disease. N. Engl. J. Med. 336:1066-1071.

34. Wojcik, C., and Di Napoli, M. 2004. Ubiquitin-proteasome system and proteasome inhibition: new strategies in stroke therapy. Stroke. 35:1506-1518.

35. Chen, Z., et al. 1995. Signal-induced site-specific phosphorylation targets I kappa B alpha to the ubiquitinproteasome pathway. Genes Dev. 9:1586-1597.

36. Amir, R.E., Iwai, K., and Ciechanover, A. 2002. The NEDD8 pathway is essential for SCF(beta$\operatorname{TrCP}$ )-mediated ubiquitination and processing of the NF-kappa B precursor p105. J. Biol. Chem. 277:23253-23259.

37. Jarrar, D., et al. 2002. Alveolar macrophage activation after trauma-hemorrhage and sepsis is dependent on NF-kappaB and MAPK/ERK mechanisms. Am. J. Physiol. Lung Cell. Mol. Physiol. 283:L799-L805.
38. Shenkar, R., et al. 1996. Hemorrhage activates NFkappa B in murine lung mononuclear cells in vivo. Am. J. Physiol. 270:L729-L735.

39. Majumdar, S., and Aggarwal, B.B. 2003. Adenosine suppresses activation of nuclear factor-kappaB selectively induced by tumor necrosis factor in different cell types. Oncogene. 22:1206-1218.

40. Read, M.A., et al. 2000. Nedd8 modification of cul-1 activates SCF(beta(TrCP))-dependent ubiquitination of IkappaBalpha. Mol. Cell. Biol. 20:2326-2333.

41. El Eter, E., Hagar, H.H., Al-Tuwaijiri, A., and Arafa, M. 2005. Nuclear factor-kappaB inhibition by pyrrolidinedithiocarbamate attenuates gastric ischemia-reperfusion injury in rats. Can. J. Physiol. Pharmacol. 83:483-492.

42. Frantz, S., et al. 2006. Absence of NF-kappaB subunit p50 improves heart failure after myocardial infarction. FASEB J. 20:1918-1920.

43. Eltzschig, H.K., et al. 2004. Endogenous adenosine produced during hypoxia attenuates neutrophil accumulation: coordination by extracellular nucleotide metabolism. Blood. 104:3986-3992.

44. Kong, T., Westerman, K.A., Faigle, M., Eltzschig, H.K., and Colgan, S.P. 2006. HIF-dependent induction of adenosine $\mathrm{A} 2 \mathrm{~B}$ receptor in hypoxia. FASEB J. 20:2242-2250.

45. Yang, D., et al. 2006. The $\mathrm{A}_{2 \mathrm{~B}}$ adenosine receptor protects against inflammation and excessive vascular adhesion. J. Clin. Invest. 116:1913-1923. doi:10.1172/JCI27933.

46. Sun, C.X., et al. 2006. Role of $\mathrm{A}_{2 \mathrm{~B}}$ adenosine receptor signaling in adenosine-dependent pulmonary inflammation and injury. J. Clin. Invest. 116:2173-2182. doi:10.1172/JCI27303.

47. Parry, G., and Estelle, M. 2004. Regulation of cullinbased ubiquitin ligases by the Nedd8/RUB ubiquitin-like proteins. Semin. Cell Dev. Biol. 15:221-229.

48. Gan-Erdene, T., et al. 2003. Identification and characterization of DEN1, a deneddylase of the ULP family. J. Biol. Chem. 278:28892-28900.

49. Mendoza, H.M., et al. 2003. NEDP1, a highly conserved cysteine protease that deNEDDylates Cullins. J. Biol. Chem. 278:25637-25643.

50. Wu, K., et al. 2003. DEN1 is a dual function protease capable of processing the $\mathrm{C}$ terminus of Nedd8 and deconjugating hyper-neddylated CUL1. J. Biol. Chem. 278:28882-28891.

51. Ibla, J.C., and Khoury, J. 2006. Methods to assess tissue permeability. Methods Mol. Biol. 341:111-117. 